\title{
The assessment of consecutive 4D-CT scans during simulation for lung stereotactic body radiation therapy patients
}

\author{
Milovan SAVANOVIC ${ }^{\mathrm{a}, \mathrm{b},{ }^{*}, \text { Bojan STRBAC }}{ }^{\mathrm{c}}$, Drazan JAROS $^{\mathrm{d}}$, Jean-Noel FOULQUIER ${ }^{\mathrm{a}}$ \\ ${ }^{a}$ Department of Radiation Oncology, Tenon Hospital, 75020 Paris, France \\ ${ }^{b}$ Faculty of Medicine, University of Paris-Saclay, 94276 Le Kremlin-Bicêtre, France \\ ${ }^{c}$ MATER Private Hospital, Physics department, Eccles Street, Dublin 7, Ireland \\ ${ }^{d}$ Affidea, International Medical Centers, Center for Radiotherapy, 78000 Banja Luka, Bosnia, and Herzegovina \\ *E-mail address: milovan_savanovic@yahoo.com
}

\begin{abstract}
Purpose: To evaluate the breathing amplitude, tumor motion, patient positioning, and treatment volumes among consecutive four-dimensional computed tomography (4D-CT) scans, during the simulation for lung stereotactic body radiation therapy (SBRT).

Material and methods: The variation and shape of the breathing amplitude, patient positioning, and treatment volumes were evaluated for 55 lung cancer patients after consecutive 4D-CT acquisitions, scanned at one-week intervals. The impact of variation in the breathing amplitude on lung tumor motion was determined for 20 patients. The gross tumor volume (GTV) was contoured from a free-breathing CT scan and at ten phases of the respiratory cycle, for both 4D-CTs (440 phases in total).

Results: Breathing amplitude decreased by 3.6 (3.4-4.9) $\mathrm{mm}$, tumor motion by 3.2 (0.4-5.0) mm while breathing period increased by 4 (2-6) s, inter-scan for 20 patients. Intra-scan variation was 4 times greater for the breathing amplitude, 5 times for the breathing period, and 8 times for the breathing cycle, comparing irregular versus regular breathing patterns for 55 patients. Using coaching, the breathing amplitude increases 3 to $8 \mathrm{~mm}$, and the breathing period 2 to 6 s. Differences in the contoured treatment volumes were less than $10 \%$ between consecutive scans. Patient positioning remained stable, with a small inter-scan difference of $1.1(0.6-1.4) \mathrm{mm}$.

Conclusion: Decreasing the inter-scan breathing amplitude decreases the tumor motion reciprocally. When the breathing amplitude decreases, the breathing period increases at inter- and intra-scan, especially during irregular breathing. Coaching improves respiration, keeping the initial shape of the breathing amplitude. Contoured treatment volumes and patient positioning were reproducible through successive scans.
\end{abstract}

Key words: lung cancer; breathing amplitude; tumor motion; 4D-CT; SBRT.

\section{Introduction}

Respiration is a gas exchange process in which the body takes oxygen and releases carbon dioxide simultaneously. During this process, all organs located in the chest and abdomen are being displaced. ${ }^{1}$ This organ motion can cause dosimetric and geometric uncertainties during treatment planning and treatment delivery, causing the target to remain under-dosed, while the surrounding healthy tissue is overdosed. ${ }^{2}$ Motion induced by respiration and/or the patient's position on the treatment couch becomes more important for the stereotactic body radiation therapy (SBRT) treatment, which uses a high dose (60 Gy for lung cancer), delivered in a few fractions (3 to 8 fractions). ${ }^{3}$ SBRT treatments need higher precision due to an escalating dose, a steep dose gradient, and adequate immobilization in order to reduce geometrical target missing. ${ }^{4}$ However, lung tumors are mobile, especially near the diaphragm. ${ }^{5}$ For lung tumors with a large motion amplitude, it is necessary to utilize motion management strategies such as the deep inspiration breath-hold (DIBH), abdominal compression, and respiratory gating radiation therapy (RGRT), to reduce treatment volume and spare the organs at risk (OARs). ${ }^{6}$ The application of these breathing techniques depends on tumor localization, the breathing amplitude, and the general state of the patient.

To reduce patient participation and to allow patients to be in the most comfortable position, the free-breathing (FB) technique was proposed with the acquirement of 4D-CTs during a simulation. ${ }^{2}$ A 4D-CT scan allows for the evaluation of the tumor motion and the motion of the OARs within the respiratory cycle, depending on the performed breathing amplitude. ${ }^{7}$ Even with irregular breathing patterns, the target shape and trajectory were also well represented using 4D-CT 
scans. ${ }^{8}$ Accurate treatment is based on precise simulation, which can be impacted by patient selection, positioning, cooperation, and/or coaching during treatment simulation. Patient respiration and positioning may affect tumor motion, decreasing the quality of CT and 4D-CT images, which makes contouring and treatment planning difficult. ${ }^{9}$ To minimize uncertainty during simulation, several studies were evaluated with results of the impact of the breathing amplitude on tumor motion, with and without coaching, between 2 to 4 successive 4D-CT scans taken before and during treatment. ${ }^{10-13}$

In this study, 2 consecutive 4D-CT scans were performed during simulation, scanned at one-week intervals. The study's aim is to evaluate inter- and intra-scan variation of the breathing amplitude, tumor motion, patient positioning and the treatment volumes. Furthermore, patients with irregular breathing patterns were coached to perform breathing as regular as possible.

\section{Materials and Methods}

\section{Patient selection}

All patients were enrolled in an Institutional Review Board approved protocol. This protocol was Health Insurance Portability and Accountability Act compliant.

The study was conducted on a consecutive cohort of 55 patients (32 males and 23 females) with small tumor size ( $\leq$ $5 \mathrm{~cm}$ ) and who underwent SBRT treatment. 52 patients had 2 consecutive 4D-CT scans and 3 patients had 3 scans. The tumor motion was evaluated for 20 patients between 2 consecutive scans (12 males and 8 females). The tumor locations depended on the lobe (10 tumors located in the upper lobe and 10 tumors located in the lower lobe) and region (12 tumors located in the central region defined as $\leq 2 \mathrm{~cm}$ from hilum and 8 tumors located in the peripheral region defined as $>2 \mathrm{~cm}$ from hilum).

\section{Data acquisition}

Using Institutional protocol, patients with small lung tumor size $(\leq 5 \mathrm{~cm})$ were immobilized in the BlueBAG BodyFIX immobilization system (Medical Intelligence, Schwabmünchen, Germany), to ensure patient positioning and to reduce inter- and intra-fraction motion. ${ }^{14}$ All patients underwent one free-breathing CT scan with a stereotactic body frame, another free-breathing CT scan without a stereotactic body frame, and one 4D-CT scan. The CT acquisition, with a slice thickness of $1.25 \mathrm{~mm}$, allowed for a better distinction of small lung tumors from OARs during delineation. ${ }^{15}$ All scans were performed with a $0.7 \mathrm{~s} /$ rotation period, $120 \mathrm{kVp}, \mathrm{mA}$ ranging from 10 to $440 \mathrm{~mA}$, using a 16 slices detector and a slice thickness of 1.25 $\mathrm{mm}$ (the beam collimation width was $20 \mathrm{~mm}$ ) and with a field of view (FOV) of $55 \mathrm{~cm}$. The tube current modulation (TCM) was turned on in order to keep image noise and image quality at a maximum constant for all examinations.
All patients were scanned on a 16 slice CT scan (GE Lightspeed, General Electric Medical Systems, Waukesha, WI), equipped with the Real-Time Positioning Management system (RPM, Varian Medical Systems, Palo Alto, CA, USA). The patient's breathing amplitude was captured with a charged coupled device (CCD) camera and the detected motion of the external marker block was located at the caudal end of the xiphoid process. The resulting respiratory curve, divided into ten phases from $0 \%$ to $90 \%$, correlated the external marker motion with the internal tumor motion, allowing the association of the images to their given phases in retrospective mode. Images were correctly associated with the phases for the regular breathing amplitude. For the irregular breathing amplitude, the association of the images to the phases lead to the appearance of the artefacts, which impacted image quality. Patients with irregular breathing amplitudes were coached to perform free-breathing with as much regular breathing as possible.

Another 4D-CT scan was performed at a one-week interval, to ensure the reproducibility of patients' positioning, inter- and intra-scan motion, and to verify tumor presence within the internal target volume (ITV) from the second scan covered by the PTV from the first scan. An image fusion between the first and second free-breathing CT scan without a stereotactic body frame was used to evaluate patient positioning using rigid bone structure (vertebra and ribs).

\section{Delineation}

In clinical routine, the delineation of the gross tumor volume (GTV) was performed in AdvantageSim (General Electric Medical Systems, Waukesha, WI, USA), on a free-breathing CT scan without a stereotactic body frame. To encompass the overall tumor motion with the ITV, the tumor motion was delineated from a 4D-CT acquisition, using a maximum intensity projection (MIP). ${ }^{16}$ Geometrical uncertainty was included in treatment planning, adding an isotropic margin of 3 $\mathrm{mm}$ to the ITV, creating the planning target volume (PTV). ${ }^{17}$ The targets' volume delineations (GTV, ITV, and PTV) were performed by a radiation oncologist and were reviewed before treatment at the tumor board meeting.

To evaluate tumor motion, a test version (T-box) of the treatment planning system (TPS) Eclipse version 13.7 (Varian Medical Systems, Palo Alto, CA, USA) was used in this study. The GTV was delineated for 20 last patients from this cohort (the phases of the first 35 patients were overwritten due to change of the Picture Archive and Communication System (PACS)), using the free-breathing CT scan without a stereotactic body frame and all ten phases (11 phases per patient), acquired with 4D-CTs. Delineation was performed by a medical physicist over all 220 phases per scan, with 440 phases in total for both 4D-CT scans. 


\section{Data analysis}

The GTV displacement through the respiratory cycle was evaluated from the center of mass, using statistical tools from DICOM images (Digital Imaging and Communications in Medicine). The overall tumor motion and patient positioning were calculated from the magnitude of a $3 \mathrm{D}$ vector represented in a three-dimensional (3D) space:

$\|\rightarrow \underset{r}{ }\|=\sqrt{x^{2}+y^{2}+z^{2}}$

Eq. 1

- $\mathrm{x}$ was the left-right (LR), $\mathrm{y}$ the anterior-posterior (AP) and $\mathrm{z}$ the craniocaudal (CC).

The breathing amplitude was calculated as a difference between the minimum and maximum position of the external marker motion during patient respiration.

The statistical analysis was performed using statistical software OriginPro version 8.6 (Northampton, MA, USA). The data were considered statistically significant at $p<0.05$. The breathing amplitude and respiratory cycle were compared between the first and second scans, using the Wilcoxon signedrank test, for all 55 patients. Also, patient positioning was evaluated between 2 scans in all directions and 3D motion. Tumor motion, the breathing amplitude, the respiratory cycle, and treatment volumes (GTV, ITV, and PTV) were compared between the first and second scans for 20 patients, using the Wilcoxon signed-rank test. The breathing amplitudes and periods were evaluated between 3 scans for 3 patients.

\section{Results}

The breathing amplitude and respiratory cycle were observed and analyzed for all 55 patients. The median value was 14.3 $(8.1-25.6) \mathrm{mm}$ vs. $11.9(7.7-22.6) \mathrm{mm}(\mathrm{p}<0.001)$, for the respiratory cycle $3.6(1.7-9.6) \mathrm{s}$ vs $.3 .4(2.5-5.5) \mathrm{s}(\mathrm{p}=0.412)$, between first and second scans. 22 patients performed regular breathing amplitudes, an example of one of the cases is shown in Figure 1a; while 33 patients performed irregular breathing amplitudes, an example of one of the cases is shown in Figure 1b. For 17 patients, who had lower or irregular breathing amplitudes, coaching increased the breathing amplitude from 3 to $8 \mathrm{~mm}$, decreasing variations in the respiratory cycle up to $4 \mathrm{~s}$. The breathing period increased from 2 to $6 \mathrm{~s}$ inter-scan, regardless of regular or irregular breathing patterns.

Intra-scan variations were observed for the breathing amplitude, respiratory cycle, and the breathing period for both, regular and irregular breathing patterns (Figure 1). The breathing amplitude variations (baseline shift) were less than 2 $\mathrm{mm}$ for regular breathing amplitudes, while irregular breathing amplitudes varied from 3 to $8 \mathrm{~mm}$. The breathing cycle varies about $1 \mathrm{~s}$ for regular versus 2 to $8 \mathrm{~s}$ for irregular breathing amplitudes. The breathing period variations were lower than 2 $\mathrm{s}$ for regular, while irregular breathing patterns varied from 3 to $10 \mathrm{~s}$.

The difference in patient positioning among consecutive scans was greater in superior-inferior direction and resulted in $0.8(0.6-1.2) \mathrm{mm}$, while the overall displacement was $1.1(0.6-$ 1.4) $\mathrm{mm}$.

The contoured volumes (GTV, ITV, and PTV) were compared between consecutive 4D-CT scans, over 20 patients. The results were presented with median values, while the results signification with p-value in Table 1.

The results of the tumor motion, breathing amplitude, and respiratory cycle were evaluated for over 20 patients, between 2 4D-CT scans (Table 2).

Between 2 consecutive 4D-CT scans, the breathing amplitude decreased by $3.6(3.4-4.9) \mathrm{mm}$, decreasing tumor motion by $3.2(0.4-5.0) \mathrm{mm}$, inter-scan (Table 2). The correlation between 2 consecutive scans was evaluated using the Pearson correlation coefficient: 0.90 for tumor motion, 0.89 for breathing amplitude, and 0.99 for the respiratory cycle.
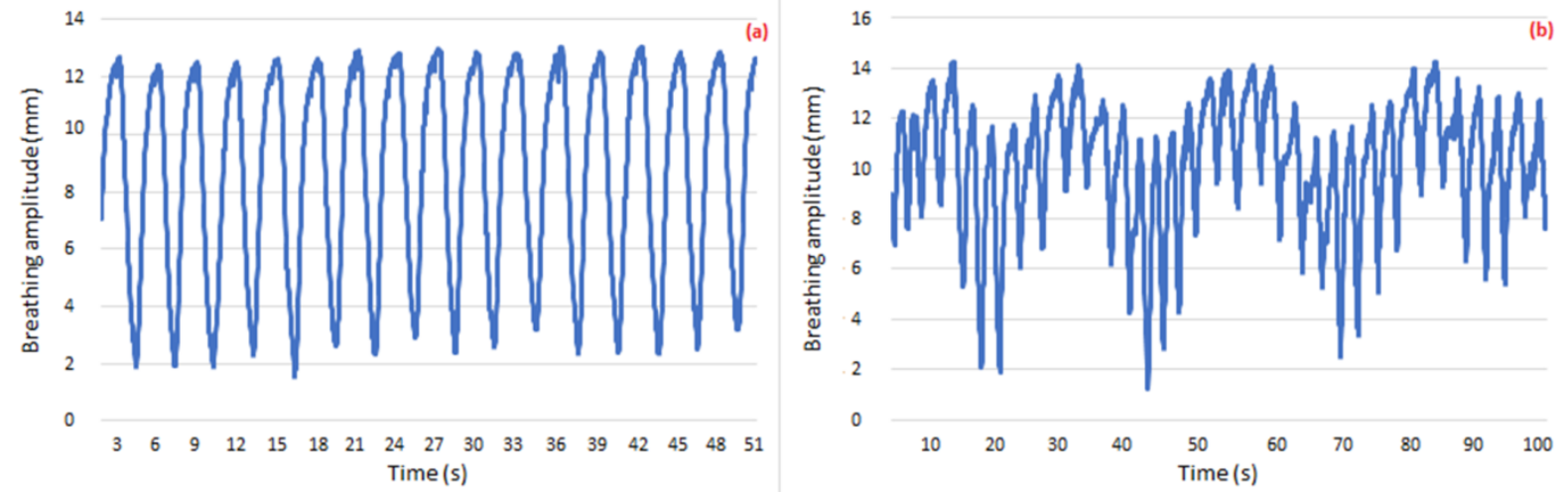

Figure 1. Examples of patient breathing with a regular (a) vs. an irregular (b) breathing amplitude 
Table 1. Comparison of the median values of contoured volumes (GTV, ITV, and PTV) between 2 4D-CT scans. The results significance was presented with p-value.

\begin{tabular}{cccc}
\hline \hline Treatment volume & First scan & Second scan & p-value \\
\hline GTV $(\mathrm{cc})$ & $2.1(0.4-16.5)$ & $2.0(0.2-15.6)$ & $=0.411$ \\
ITV $(\mathrm{cc})$ & $3.8(0.6-19.8)$ & $3.8(0.4-19.2)$ & $=0.902$ \\
PTV $(\mathrm{cc})$ & $10.3(2.8-38.3)$ & $11.2(1.6-38)$. & $<0.0001$ \\
\hline \hline
\end{tabular}

Abbreviation: GTV - gross tumor volume, ITV - internal target volume, PTV - planning target volume

Table 2. Comparison of the median values and their range of tumor motion, breathing amplitude and respiratory cycle between 2 consecutive 4D-CT scans.

\begin{tabular}{cccc}
\hline \hline Patient parameters & First scan & Second scan & p-value \\
\hline Tumor motion $(\mathrm{mm})$ & $12.0(0.9-24.0)$ & $8.8(0.5-19.0)$ & $=0.02$ \\
Breathing amplitude $(\mathrm{mm})$ & $14.3(6.7-22.6)$ & $10.7(3.3-17.7)$ & $<.0001$ \\
Respiratory cycle (s) & $3.5(2.4-9.2)$ & $3.4(2.4-8.2)$ & $<0.0001$ \\
\hline \hline
\end{tabular}
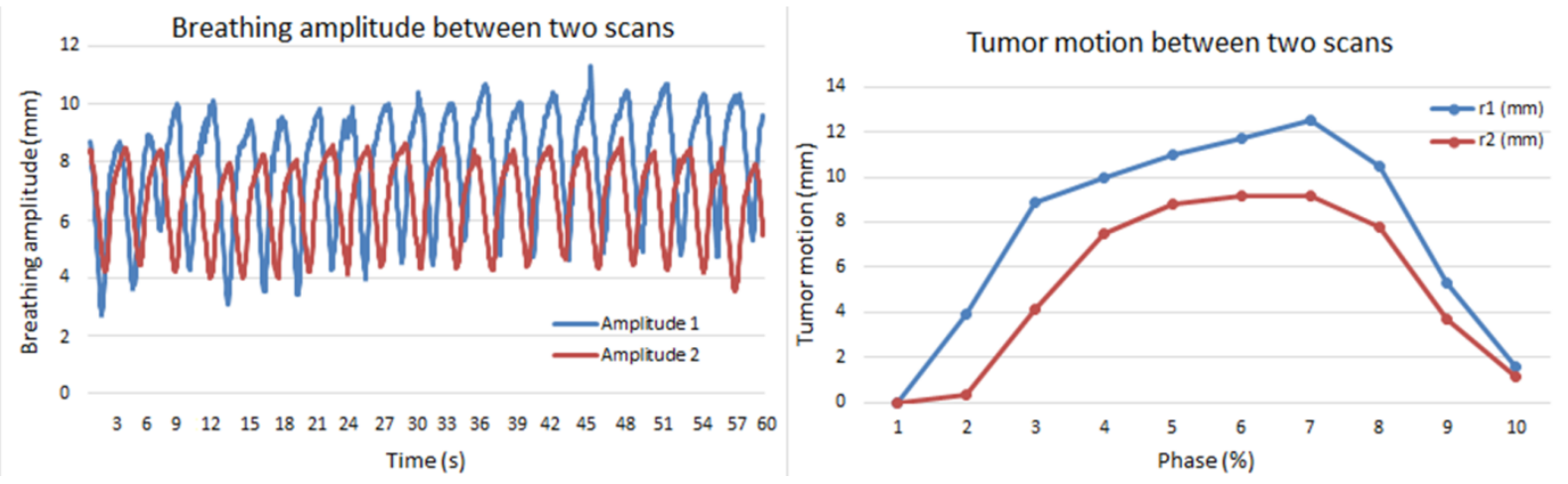

Figure 2. Comparison of the breathing amplitude and tumor motion between 2 scans for an SBRT patient with a small tumor size

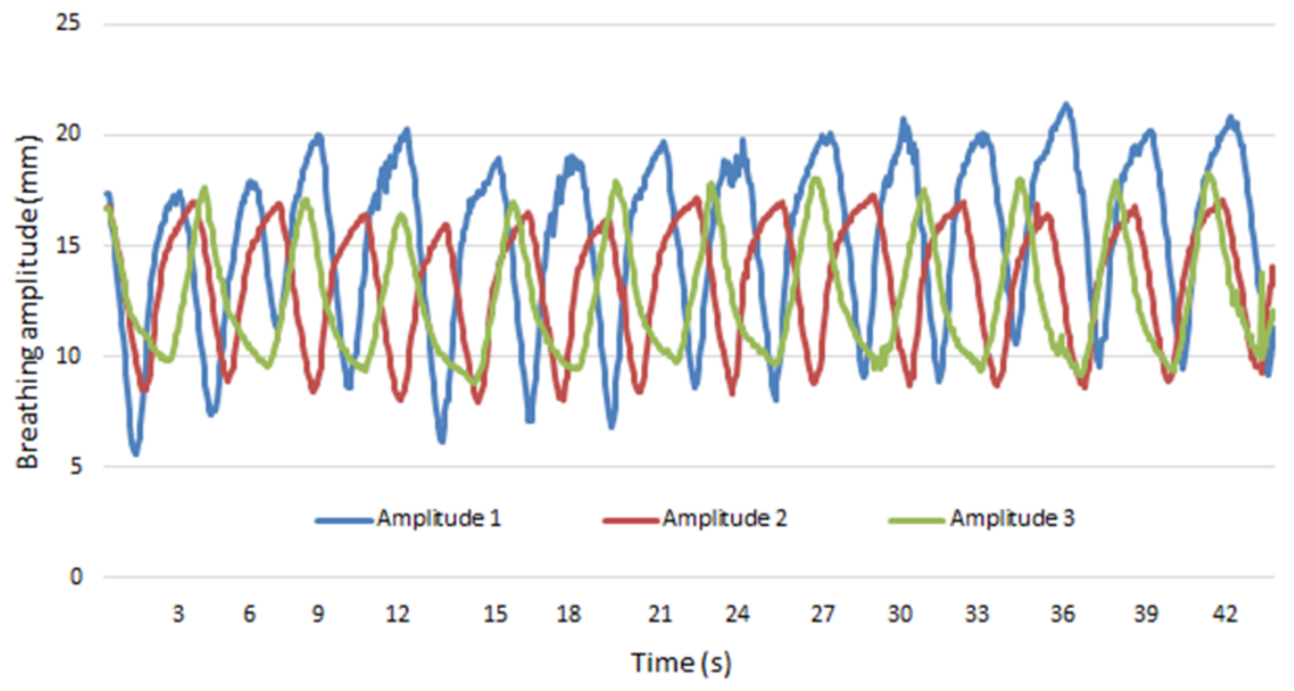

Figure 3. The shape of the breathing amplitude, obtained over 3 consecutive 4D-CT scans, is presented with a blue respiratory curve (data from the first 4D-CT), a red respiratory curve (data from the second 4D-CT) and a green respiratory curve (data from the third 4D-CT scan)

The difference between the amplitude of the tumor motion across the 2 scans is shown in Figure 2. Figure 2 shows decreasing in the breathing amplitude and amplitude of tumor motion between 2 scans (an example of one of the cases), where the blue line represents data from the first 4D-CT scan and the red line represents data from the second 4D-CT scan.

Variation in the breathing amplitude over 3 acquisitions are presented in Figure 3. 
Table 3. Evaluation of the breathing amplitude and period from 3 patients and over 3 4D-CT scans.

\begin{tabular}{|c|c|c|c|c|c|c|}
\hline \multirow{2}{*}{ 4D-CT scan } & \multicolumn{2}{|c|}{ Patient 1} & \multicolumn{2}{|c|}{ Patient 2} & \multicolumn{2}{|c|}{ Patient 3} \\
\hline & Amplitude (mm) & Period (s) & Amplitude (mm) & Period (s) & Amplitude (mm) & Period (s) \\
\hline First scan & 11.5 & 3.2 & 16.4 & 4.1 & 22.6 & 3.0 \\
\hline Second scan & 10.8 & 4.3 & 14.2 & 6.4 & 14.8 & 4.4 \\
\hline Third scan & 9.4 & 5.4 & 11.8 & 8.3 & 11.1 & 6.1 \\
\hline
\end{tabular}

Abbreviation: 4D-CT-four-dimensional computed tomography

The results for the 3 breathing amplitudes and periods were presented in Table 3 for all 3 patients.

From the results presented in Table 3, the breathing amplitudes decreased inter-scan for $0.7 \mathrm{~mm}, 2.2 \mathrm{~mm}$ and 7.8 $\mathrm{mm}$ between the first and second scans; and $1.4 \mathrm{~mm}, 2.4 \mathrm{~mm}$ and $3.7 \mathrm{~mm}$ between the second and third scan, causing a longer breathing period, which ranged from 2 to $4 \mathrm{~s}$.

\section{Discussion}

Successive 4D-CT scans were performed for 55 patients with small lung cancer. The variations in the breathing amplitudes were found within the inter-patient cohort. These variations among patients depend on the general state of the patient, including their lungs' physiology, respiratory problems, pain and/or fear of irradiation. Patients performed more irregular breathing amplitudes (60\%) than regular (40\%). For regular breathing amplitudes, the variation in the breathing cycle was less than $5 \%$ within the respiratory cycle, while it increased up to $32 \%$ for irregular breathing amplitudes. Inter- and intra-scan variations were found in the breathing amplitude, breathing cycle, and the breathing period within the respiratory cycle. The inter-scan variation in the breathing amplitude depends on initial patient respiration and can vary between several millimeters (from 1 to $8 \mathrm{~mm}$ ). With an evaluated variability of 4D-CT patient models, Sonke et al. found that the mean value of the day-to-day breathing variation was $3.9 \mathrm{~mm} .^{18}$

Coaching improved the breathing amplitude (in 30\% of patients), which became more regular, keeping its initial shape, regardless of regular or irregular breathing patterns. From the study of Hau et al., the amplitude variation in the respiratory cycle can be minimized using audio coaching. ${ }^{19}$ Kini et al. found that variability in the amplitude and frequency of the breathing cycle improves after patient coaching. ${ }^{10}$ George et al. demonstrated that coaching, complemented with audio and visual guidance, impacted the stability and reproducibility of the patient's breathing. ${ }^{20}$ Based on the study by Goossens et al., the regularity of a patient's breathing can increase using audio coaching accompanied with real-time visual feedback. ${ }^{11}$

Inter-fraction patient positioning is crucial in SBRT treatments. The negligible difference in the results obtained during patient positioning between 2 4D-CT scans approves the utilization of the BlueBAG BodyFIX immobilization system, which decreased patient motion during the SBRT treatment. Hubi et al. reported that the BlueBAG BodyFIX immobilization system is the most suitable for obtaining better patient positioning, but also the most comfortable for patients. ${ }^{14}$ In the Guckenberger et al. study, a patient motion was evaluated using stereotactic body frame (SBF, Elekta, Crawly, United Kingdom), and the study obtained similar results for the average $(1 \mathrm{~mm})$ and maximum patient motion $(1.5 \mathrm{~mm})$, as is the case with this study. ${ }^{12}$

Using the SBRT treatment, precise delineation allows better coverage of the target, decreasing the dose to the organ at risk. Comparing the contoured treatment volumes inter-scan, small differences were found for the GTV (2.4\%) and PTV (8.7\%), while the ITV volume was identical between 2 scans. These small differences in volume were negligible and confirm that the ITV and PTV margins are fairly wide to absorb overall tumor motion inter- and intra-faction. In the Van der Geld et al. study, generated target volumes had similar differences $(1.4 \pm 1.0 \mathrm{~mm})$ between the center of mass of 2 PTVs for similar dosimetric coverage from a single 4D-CT without coaching, rather than what was achieved using 2 consecutive scans. ${ }^{21}$

The impact of the breathing amplitude variation on lung tumor motion was evaluated for twenty patients, from 2 consecutive 4D-CT scans. The differences between the 2 scans were statistically significant, for $\mathrm{p}<0.05$ (as presented in Table 2). We found that the breathing amplitude decreased by $25.2 \%$ $(4.8 \%-50.7 \%)$, while the tumor motion decreased by $26.7 \%$ $(2.3 \%-56.7 \%)$. A greater difference was found in this study, compared to the study of Goossens et al., where the breathing amplitude (16.3\%) and tumor motion $(10.7 \%)$ were evaluated between successive 4D-CTs using audio-visual coaching [11]. Michalski et al. reported that the inter-fraction target motion reproducibility seems to be present in $87 \%$ of cases (22 patients) between consecutive 4D-CT scans. ${ }^{13}$

This study also found that when the breathing amplitude decreases, the breathing period increases and becomes longer by $17 \%(12.6 \%-21.4 \%)$, with a small change in the breathing cycle by $6.7 \%(0 \%-14.7 \%)$ inter-scan, for all patients. This can be explained by changes in patient conduct during and after the first scan since patients became more familiar with the machine and equipment in radiotherapy and therefore became more relaxed and convinced in the continuation of their treatment.

3 patients went through 3 4D-CT acquisitions, due to different problems between scans, such as respiration with irregular breathing patterns, coughing, and pain in the arms and shoulders, which compelled us to reposition and rescan the patient in order to ensure high image quality and precise treatment planning. We found that the breathing amplitude decreased inter-scan $(6 \%, 13 \%$, and $35 \%$ between the first and 
second scan and $13 \%, 17 \%$, and $25 \%$ between the second and third scan), while the breathing period became longer (from $25.6 \%$ to $50.8 \%$ ). The breathing amplitude decreased constantly during inter-scan, regardless of regular or irregular breathing amplitudes. This can have an impact on treatment choice (RGRT treatment based on amplitude), where the baseline shift must be changed and adapted inter-fraction (Figure 1b). On the other hand, despite the variations in the breathing amplitude, we can use RGRT treatment based on phase, knowing that the breathing cycle remains in a constant form (ten phases from $0 \%$ to $90 \%$ ) peak-to-peak. Korreman et al. reported that inter-fraction variation in patients' breathing patterns can impact the RGRT technique, evaluating margin reduction using the RGRT treatment combined with respiratory correlated image guidance. $^{22}$

\section{Conclusion}

The consecutive 4D-CT scans during the simulation for lung SBRT patients were investigated. The inter-patient cohort scans showed that the breathing amplitude decreases inter-scan, decreasing tumor motion reciprocally. When the breathing amplitude decreases, the breathing period increases and it becomes longer.
The intra-scan showed variation in the breathing amplitude and breathing period, especially during irregular breathing. Coaching is recommended to decrease variation in the breathing amplitude and to ensure stability, reproducibility, and regularity in patients' breathing.

Despite the decrease in the breathing amplitude and a longer breathing period, the shape of the breathing amplitude does not change, remaining in the initial shape for regular and irregular breathing patterns. A longer breathing period can decrease treatment time for treatment based on the RGRT. However, a regular breathing amplitude appears to be an excellent prediction factor for the treatment of lung cancer patients using the RGRT technique, especially RGRT based on amplitude.

Using an immobilization system during simulation and treatment delivery decreases uncertainties due to patient positioning, allowing precise and reproducible inter-fraction positioning. Contouring treatment volumes was reproducible among successive scans, covering tumor motion with the prescribed margin and allowing for precise and safe treatment delivery to the target.

\section{References}

1. Liauw SL, Connell PP, Weichselbaum RR. New paradigms and future challenges in Radiation Oncology: An Update of Biological Targets and Technology. Sci Transl Med. 2013;5(173): 173sr2.

2. Keall PJ, Mageras GS, Balter JM, et al. The management of Respiratory Motion in Radiation Oncology report of AAPM Task Group 76. Med Phys. 2006;33(10):3874-3900.

3. Haasbeek CJ, Lagerwaard FJ, Slotman BJ, Senan S. Outcomes of stereotactic ablative radiotherapy for centrally located early-stage lung cancer. J Thorac Oncol. 2011 Dec;6(12):2036-43.

4. Hoffman D, Dragojević I, Hoisak J, Hoopes D, Manger R. Lung Stereotactic Body Radiation Therapy (SBRT) dose gradient and PTV volume: a retrospective multi-center analysis. Radiat Oncol. 2019 Sep 3;14(1):162.

5. Wang Y, Bao Y, Zhang L et al. Assessment of respiration-induced motion and its impact on treatment outcome for lung cancer. Biomed Res Int. 2013;2013:872739.

6. Molitoris JK, Diwanji T, Snider JW 3rd et al. Advances in the use of motion management and image guidance in radiation therapy treatment for lung cancer. J Thorac Dis. 2018 Aug;10(Suppl 21):S2437-S2450.

7. Bai T, Zhu J, Yin Y, Lu J, Shu H, Wang L, Yang B. How does four-dimensional computed tomography spare normal tissues in nonsmall cell lung cancer radiotherapy by defining internal target volume? Thoracic Cancer. 2014 Nov;5(6):537-542.

8. Aznar MC, Persson GF, Kofoed IM, Nygaard DE, Korreman SS. Irregular breathing during 4DCT scanning of lung cancer patients: is the midventilation approach robust? Phys Med 2014;30:69-75.

9. Brandner ED, Chetty IJ, Giaddui TG, Xiao Y, Huq MS. Motion management strategies and technical issues associated with stereotactic body radiotherapy of thoracic and upper abdominal tumors: A review from NRG oncology. Med Phys. 2017;44(6):25952612.

10. Kini VR, Vedam SS, Keall PJ, Patil S, Chen C, Mohan R. Patient training in respiratory-gated radiotherapy. Med Dosim. 2003 Spring; 28(1):7-11.

11. Goossens S, Senny F, Lee JA, Janssens G, Geets X. Assessment of tumor motion reproducibility with audio-visual coaching through successive 4D CT sessions. J Appl Clin Med Phys 2014; 15: 4332.

12. Guckenberger M, Wilbert J, Meyer J, Baier K, Richter A, Flentje M. Is a Single Respiratory Correlated 4D-CT Study Sufficient for Evaluation of Breathing Motion? Int J Radiat Oncol Biol Phys. 2007 Apr 1; 67(5):1352-59.

13. Michalski D, Sontag M, Li F et al. Four-dimensional Computed Tomography-Based Interfractional Reproducibility Study of Lung Tumor Intrafractional Motion. Int J Radiat Oncol Biol Phys. 2008 Jul 1; 71(3):714-24. 
14. Hubie C, Shaw M, Bydder S et al. A rondomised comparison of three different immobilisation devices for thoracic and abdominal cancers. J Mec Radiat Sci. 2017 Jun; 64(2):90-96.

15. Fischbach F, Knollmann F, Griesshaber V, Freund T, Akkol E, Felix R. Detection of pulmonary nodules by multislice computed tomography: improved detection rate with reduced slice thickness. Eur Radiol. 2003 Oct;13(10):2378-83.

16. Radiation therapy oncology group. Lung cancer atlas for radiation therapy planning: consensus definitions. http://www.rtog.org/CoreLab/

17. Li Y, Ma JL, Chen X, Tang FW, Zhang XZ. 4DCT and CBCT based PTV margin in Stereotactic Body Radiotherapy(SBRT) of nonsmall cell lung tumor adhered to chest wall or diaphragm. Radiat Oncol. 2016; 11: 152.

18. Sonke JJ, Lebesque J, van Herk M. Variability of four-dimensional computed tomography patient models. Int J Radiat Oncol Biol Phys. 2008 Feb 1; 70(2):590-8.

19. Hau E, Rains M, Pham T, Muirhead R, Yeghiaian Alvandi R. Potential benefits and pitfalls of respiratory-gated radiotherapy in the treatment of thoracic malignancy. Asia Pac J Clin Oncol. 2014 Jun; 10(2):e13-20.

20. George R, Chung TD, Vedam SS, et al. Audio-visual biofeedback for respiratory-gated radiotherapy: impact of audio instruction and audio-visual biofeedback on respiratory-gated radiotherapy. Int J Radiat Oncol Biol Phys 2006; 65: 924-33.

21. van der Geld YG, Lagerwaard FJ, van Sörnsen de Koste JR, Cuijpers JP, Slotman BJ, Senan S. Reproducibility of target volumes generated using uncoached 4-dimensional CT scans for peripheral lung cancer. Radiat Oncol. 2006; 1:43.

22. Korreman SS, Juhler-Nøttrup T, Boyer AL. Respiratory gated beam delivery cannot facilitate margin reduction, unless combined with respiratory correlated image guidance. Radiother Oncol. 2008 Jan; 86(1):61-8. 